\title{
Electrodynamics of the Joule-Lenz Law Applied to the Energy Emission Done by a Free Electron or Harmonically-Oscillating Microparticle
}

\author{
Stanisław Olszewski \\ Institute of Physical Chemistry, Polish Academy of Sciences, Warsaw, Poland \\ Email: olsz@ichf.edu.pl
}

How to cite this paper: Olszewski, S. (2018) Electrodynamics of the Joule-Lenz Law Applied to the Energy Emission Done by a Free Electron or Harmonically-Oscillating Microparticle. Journal of Quantum Information Science, 8, 121-130.

https://doi.org/10.4236/jqis.2018.83008

Received: July 2, 2018

Accepted: August 19, 2018

Published: August 22, 2018

Copyright $\odot 2018$ by author and Scientific Research Publishing Inc. This work is licensed under the Creative Commons Attribution International License (CC BY 4.0).

http://creativecommons.org/licenses/by/4.0/

\section{Open Access}

\begin{abstract}
We demonstrate that the intensity of the energy emission obtained from the Joule-Lenz law applied to the case of a single free-electron particle or a harmonic oscillator does not depend on the change of size of the corresponding energy interval $(\Delta E)$ and time interval $(\Delta t)$ because the ratio of $\Delta E$ to $\Delta t$ representing the emission rate remains constant. For a free electron, this property holds on condition the calculations of $\Delta E$ and $\Delta t$ refer to the states having a sufficiently large quantum index $n$.
\end{abstract}

\section{Keywords}

Energy Emission Intensity, Joule-Lenz Law, Simple Quantum Systems

\section{Introduction}

The kind of electrodynamics presented in the paper seems to be not studied enough in the former approaches to the energy emission. Two simple physical cases-of a free particle in the potential box and a linear harmonic oscillatorare mainly considered. The formalism applied to them is based on the JouleLenz equation used predominantly before in a classical approach to a dissipated energy [1] [2]. But next it became evident that the electrodynamical apparatus of the currents intensity and their resistance entering the Joule-Lenz theory could be applied also to the electron microparticles [3].

The present calculations begin with free electrons whose behaviour in a potential box is well known. With the electron-electron interaction neglected and the box size limited to an interval of a straight line having infinite potential walls, the energy quanta in the box are [4]: 


$$
E_{n}=\frac{n^{2} h^{2}}{8 m L^{2}}
$$

where

$$
n=1,2,3, \cdots,
$$

$m$ is the electron mass, $L$ is the box length.

The energy emission is an effect of the change of some number $n$ in (1) into a lower one. For the sake of convenience let us assume that some number $n+1$ is changed into $n$ giving the emitted energy equal to

$$
\Delta E=E_{n+1}-E_{n}=\frac{(n+1)^{2}-n^{2}}{8 m L^{2}} h^{2} .
$$

Now the main problem concerns the time interval $\Delta t$ which is connected with the process represented by (3), since a knowledge of $\Delta t$ combined with $\Delta E$ in (3) leads to the intensity of the energy emission. In quantum mechanics this intensity is obtained on a probabilistic way based on the calculation of the matrix elements produced by the wave functions entering the beginning and end quantum states involved in the emission process, combined also with the appropriate operators [5] [6].

This is a tedious way which we try to replace by a more simple semiclassical approach based on the Joule-Lenz law:

$$
\frac{\Delta E}{\Delta t}=R i^{2}
$$

where $R$ is the electric resistance and $i$ the current intensity supplied by the electron particle. In effect we obtain the ratio of the emitted energy $\Delta E$ and time $\Delta t$ of the occurence of $\Delta E$ as a function of $R$ and $i$. The resistance is

$$
R=\frac{V}{i},
$$

where we put

$$
V=\frac{\Delta E}{e}
$$

and

$$
i=e / T
$$

where $e$ is the electron charge and $T$ is the time period connected with the electron current on level $n$. We find below that the same $T$ does approach the time interval necessary for transition between the levels $n+1$ and $n$.

The formulae (5)-(7) complete the formalism necessary to calculate (4) on condition we have the time period $T=T_{n}$. For a free electron located in state $n$ this parameter should satisfy the equation for the electron velocity in state $n$ :

$$
v_{n}=\frac{2 L}{T_{n}}=\left(\frac{2 E_{n}}{m}\right)^{1 / 2}
$$

obtained by assuming that the free-electron energy (1) is equal to the kinetic 
energy given by the formula

$$
\frac{m v_{n}^{2}}{2}=E_{n} .
$$

A substitution of (1) into (8) provides us with

$$
\frac{2 L}{T_{n}}=\frac{n h}{2 m L}
$$

from which

$$
T_{n}=\frac{4 m L^{2}}{n h} .
$$

\section{Electric Resistance $R$ and the Emission Rate of Energy in Equation (4)}

In this and next Sections we apply systematically formula (4) and complementary formulae (5)-(7). The aim of calculations is to present the emission intensity (4) for the case of the energy difference $\Delta E$ between the quantum levels given in (3), and next for energy differences

$$
\begin{aligned}
& E_{n+2}-E_{n}, \\
& E_{n+3}-E_{n}, \\
& E_{n+4}-E_{n},
\end{aligned}
$$

and

$$
E_{n+5}-E_{n} .
$$

In the first step, on the basis of (5)-(7) and (10a), we have

$$
R=\frac{V}{i}=\frac{\Delta E}{e} \frac{T_{n}}{e}=\frac{(n+1)^{2}-n^{2}}{8 m L^{2} e} h^{2} \frac{4 m L^{2}}{n h e} \cong \frac{n h^{2}}{4 m L^{2} e} \frac{4 m L^{2}}{n h e}=\frac{h}{e^{2}},
$$

on condition $n$ is large enough to satisfy

$$
2 n \gg 1 .
$$

A characteristic point is that the result in (11) does not depend on $n$. Evidently this result is identical with the resistance

$$
R=\frac{h}{e^{2}}
$$

characteristic for the integer quantum Hall effect; see e.g. [7].

A posteriori the result obtained in (11) justifies that

$$
T_{n}=\Delta t
$$

where $\Delta t$ is the time interval applied on the left side of (4). For, a substitution of

$$
\Delta E=\Delta E_{n} \cong \frac{n h^{2}}{4 m L^{2}}
$$

entering (11) into (4) gives

$$
\frac{\Delta E}{\Delta t} \cong \frac{n h^{2}}{4 m L^{2}} \frac{1}{\Delta t}=\frac{R e^{2}}{T_{n}^{2}}=\frac{h}{e^{2}} \frac{e^{2}}{T_{n}^{2}}=\frac{h}{T_{n} T_{n}}=\frac{n h^{2}}{4 m L^{2}} \frac{1}{T_{n}}=\frac{n^{2} h^{3}}{16\left(m L^{2}\right)^{2}} .
$$


The one-by-last step of (16) obtained from (10a) compared with the second step entering (16) leads to the equation

$$
\frac{n h^{2}}{4 m L^{2}} \frac{1}{\Delta t}=\frac{n h^{2}}{4 m L^{2}} \frac{1}{T_{n}}
$$

which is equivalent to the formula (14).

\section{Resistance $R$ and the Transition Time of Electron in Case of the Emission Energy $\Delta E=\Delta E_{n+1}>\Delta E_{n}$}

Let us assume here that

$$
\Delta E=\Delta E_{n+1}=E_{n+2}-E_{n}=\frac{(n+2)^{2}-n^{2}}{8 m L^{2}} h^{2} \cong \frac{n h^{2}}{2 m L^{2}}=e V
$$

and

$$
\Delta t=T_{n}+T_{n+1}=\frac{4 m L^{2}}{n h}+\frac{4 m L^{2}}{(n+1) h} \cong \frac{8 m L^{2}}{n h}
$$

where the second T-component in (19) is the time necessary for transition between the levels $n+2$ and $n+1$ :

$$
T_{n+1}=\frac{4 m L^{2}}{(n+1) h}
$$

see (10a).

In this case the resistance $R$ becomes

$$
R=\frac{V}{i}=\frac{n h^{2}}{2 m L^{2} e} \frac{\Delta t}{e}=\frac{n h^{2}}{2 m L^{2} e^{2}} \frac{8 m L^{2}}{n h}=\frac{4 h}{e^{2}} .
$$

We find that the value of $R$ is four times larger than that obtained in (11). The condition for $n$ satisfied in calculating $R$ is here

$$
4 n \gg 4 \text { or } n \gg 1 \text {. }
$$

We can check the Joule-Lenz equation (4) by substituting into it the results obtained in (18)-(21):

$$
\frac{\Delta E}{\Delta t}=\frac{n h^{2}}{2 m L^{2}} \frac{n h}{8 m L^{2}}=\frac{4 h}{e^{2}} \frac{e^{2}}{(\Delta t)^{2}}=\frac{4 h}{e^{2}} \frac{e^{2} n^{2} h^{2}}{\left(8 m L^{2}\right)^{2}}=\frac{4}{64} \frac{n^{2} h^{3}}{\left(m L^{2}\right)^{2}}=\frac{1}{16} \frac{n^{2} h^{3}}{m^{2} L^{4}} .
$$

Evidently both sides of (4) fit together on condition the formula (19) for $\Delta t$ is consequently applied in calculating the current intensity $i$.

\section{Next Step of the Extension of $\Delta E, R$ and $\Delta t$ Applied to the Joule-Lenz Law}

In this step we take into account

$$
\Delta E=\Delta E_{n+2}=E_{n+3}-E_{n}=\frac{(n+3)^{2}-n^{2}}{8 m L^{2}} h^{2} \cong \frac{6 n}{8 m L^{2}} h^{2}=\frac{3}{4} \frac{n h^{2}}{m L^{2}}
$$

for which the formula 


$$
6 n \gg 9
$$

is assumed. The subscript $n+2$ of $\Delta E$ in (24) indicates the interval $\Delta E$ larger by one step than that having subscipt $n+1$ in Sec. 3; see (18).

On the other side we apply

$$
\Delta t=T_{n}+T_{n+1}+T_{n+2}=\frac{4 m L^{2}}{n h}+\frac{4 m L^{2}}{(n+1) h}+\frac{4 m L^{2}}{(n+2) h} \approx \frac{12 m L^{2}}{n h}
$$

valid for large $n$. In effect the resistance $R$ becomes

$$
R=\frac{V}{i}=\frac{\Delta E}{e^{2}} \Delta t=\frac{3 n h^{2}}{4 m L^{2}} \frac{1}{e^{2}} \frac{12 m L^{2}}{n h}=\frac{9 h}{e^{2}} .
$$

With the aid of the above formulae a next check of the application of the Joule-Lenz law in (4) can be done:

$$
\frac{\Delta E}{\Delta t}=\frac{3}{4} \frac{n h^{2}}{m L^{2}} \frac{n h}{12 m L^{2}}=\frac{9 h}{e^{2}} \frac{e^{2}}{(\Delta t)^{2}}=9 h \frac{n^{2} h^{2}}{144\left(m L^{2}\right)^{2}}=\frac{1}{16} \frac{n^{2} h^{3}}{\left(m L^{2}\right)^{2}} .
$$

\section{Further Extensions of the Formula (4) to Large $\Delta E$ and $\Delta t$}

These extensions demonstrate us a constant result of the emission rate $\Delta E / \Delta t$ obtained for different separations between the quantum levels $n+q$ and $n$. Let us consider two examples of $\Delta E$ and $\Delta t$ being larger than applied before. The first one is

$$
\Delta E=\Delta E_{n+3}=E_{n+4}-E_{n}=\frac{(n+4)^{2}-n^{2}}{8 m L^{2}} h^{2} \cong \frac{8 n h^{2}}{8 m L^{2}}=\frac{n h^{2}}{m L^{2}}
$$

valid on condition

$$
8 n \gg 16 \text { or } n \gg 2 .
$$

The $\Delta t$ connected with $\Delta E$ in (29) is

$$
\begin{aligned}
\Delta t & =T_{n}+T_{n+1}+T_{n+2}+T_{n+3} \\
& =\frac{4 m L^{2}}{n h}+\frac{4 m L^{2}}{(n+1) h}+\frac{4 m L^{2}}{(n+2) h}+\frac{4 m L^{2}}{(n+3) h} \approx \frac{16 m L^{2}}{n h} .
\end{aligned}
$$

Both formulae (29) and (31) give for the left side of (4):

$$
\frac{\Delta E}{\Delta t}=\frac{n h^{2}}{m L^{2}} \frac{n h}{16 m L^{2}}=\frac{n^{2} h^{3}}{16\left(m L^{2}\right)^{2}} .
$$

On the right-hand side of (4) we obtain

$$
R=\frac{V}{i}=\frac{V \Delta t}{e}=\frac{\Delta E \Delta t}{e^{2}}=\frac{n h^{2}}{m L^{2}} \frac{1}{e^{2}} \frac{16 m L^{2}}{n h}=16 \frac{h}{e^{2}}
$$

and

$$
i^{2}=\frac{e^{2}}{(\Delta t)^{2}}=\frac{e^{2}}{\left(16 m L^{2}\right)^{2}} n^{2} h^{2} .
$$

Therefore the product of (33) and (34) gives 


$$
R i^{2}=16 \frac{h}{e^{2}} \frac{e^{2}}{\left(16 m L^{2}\right)^{2}} n^{2} h^{2}=\frac{n^{2} h^{3}}{16\left(m L^{2}\right)^{2}}
$$

which is a result equal to that obtained in (32).

As the end step let us take

$$
\Delta E=\frac{(n+5)^{2}-n^{2}}{8 m L^{2}} h^{2} \cong \frac{10 n h^{2}}{8 m L^{2}}=\frac{5}{4} \frac{n h^{2}}{m L^{2}} .
$$

The resistance corresponding to (36) becomes

$$
R=\frac{V}{i}=\frac{\Delta E}{e^{2}} \Delta t=\frac{1}{e^{2}} \frac{5}{4} \frac{n h^{2}}{m L^{2}} \frac{20 m L^{2}}{n h}=25 \frac{h}{e^{2}},
$$

since $\Delta t$ being an extension of (31) appropriate to transition energy (36) is

$$
\Delta t \approx \frac{20 m L^{2}}{n h} \text {. }
$$

In the next step

$$
i^{2}=\frac{e^{2}}{(\Delta t)^{2}}=\frac{e^{2}}{\left(20 m L^{2}\right)^{2}} n^{2} h^{2},
$$

so by combining $i^{2}$ in (39) with $R$ from (37) we obtain

$$
R i^{2}=25 \frac{h}{e^{2}} \frac{e^{2}}{\left(20 m L^{2}\right)^{2}} n^{2} h^{2}=\frac{25}{400} \frac{n^{2} h^{3}}{\left(m L^{2}\right)^{2}}=\frac{1}{16} \frac{n^{2} h^{3}}{\left(m L^{2}\right)^{2}} .
$$

On the other hand the ratio of (36) and (38) gives

$$
\frac{\Delta E}{\Delta t}=\frac{5}{4} \frac{n h^{2}}{m L^{2}} \frac{n h}{20 m L^{2}}=\frac{1}{16} \frac{n^{2} h^{3}}{\left(m L^{2}\right)^{2}}
$$

which is a result identical with that obtained in (40).

We conclude this section by a remark that the emission rate of energy due to a free electron enclosed in a one-dimensional potential box examined semiclassically with the aid of the Joule-Lenz law does not depend on the separation between states

$$
n+q \text { and } n
$$

on condition we have satisfied the relation

$$
n \gg q .
$$

It is worth to be noted that the emission rate of a free particle energy multiplied by $(\Delta t)^{2}$ gives a result independent of parameters $n$ and $L$. For example by multiplying (41) by the square value of the time interval in (38) we obtain

$$
\Delta E \Delta t=25 h .
$$

It is easy to see that a general formula of that kind will be

$$
\Delta E \Delta t=q^{2} h
$$

when the intervals $\Delta E$ and $\Delta t$ suitable for a given $q$ are taken into account. 


\section{Emission Rate of Energy for the Harmonic Oscillator}

In general our aim is to consider the emission rate of energy

$$
\Delta E=E_{n+1}-E_{1}=n h v=n h \frac{1}{T}
$$

of a linear harmonic oscillator due to the energy change, say, between the quantum levels $n+1$ and $n=1$. The frequency $v$ of the oscillator refers to the time period $T$ of the oscillation:

$$
v=1 / T
$$

We apply the Joule-Lenz law beginning with transition

$$
\Delta E=E_{n+1}-E_{n}=h v .
$$

The resistance $R$ entering the law is

$$
R=\frac{V}{i}=\frac{\Delta E}{e i}=\frac{h v}{e^{2}} T=\frac{h}{e^{2}}
$$

since

$$
i=e / T \text {. }
$$

Evidently the $R$ in (46) is identical with that obtained for the lowest free-electron transition in (11). In effect of (45)-(47) the right-hand side of the Joule-Lenz law (4) becomes

$$
R i^{2}=\frac{h}{e^{2}} \frac{e^{2}}{T^{2}}=\frac{h}{T^{2}} .
$$

The left-hand side of (4) in the oscillator case is

$$
\frac{\Delta E}{\Delta t}=\frac{h v}{\Delta t}=\frac{h}{T \Delta t} .
$$

The requirement of equality between (48) and (49) gives

$$
\frac{h}{T \Delta t}=\frac{h}{T^{2}}
$$

or

$$
\Delta t=T
$$

In effect the time rate of the energy change is

$$
\frac{\Delta E}{\Delta t}=\frac{h}{T^{2}}=h v^{2} \text {. }
$$

In case of the energy separation done by two quantum levels, namely

$$
\Delta E=E_{n+2}-E_{n}=E_{n+2}-E_{n+1}+E_{n+1}-E_{n},
$$

the time interval $\Delta t$ required to attain the difference (52) is twice the interval $T$ necessary to attain the difference between two neighbouring levels [see (50a)]:

$$
\Delta t=\Delta t_{2}=T+T=2 T \text {. }
$$

This means that the emission intensity is

$$
\frac{\Delta E}{\Delta t}=\frac{2 h v}{2 T}=\frac{h v}{T}=h v^{2},
$$


so the intensity for the case of the energy difference (52) is the same as intensity obtained in the case of (45); see (51).

Let us examine still the right-hand side of the Joule-Lenz law presented in (4) for $\Delta E$ in (52). In this case

$$
R=\frac{\Delta E}{e i}=\frac{\Delta E}{e} \frac{\Delta t}{e}=\frac{2 h \nu}{e^{2}} 2 T=4 \frac{h}{e^{2}}
$$

which is the same $R$ as obtained in (18). The calculation indicates that $R$ in two different physical systems-free particle in the box and harmonic oscillator-can be the same on condition the separation number $q$ between the quantum levels $n+q$ and $n$ is unchanged.

In general the time interval necessary to make a travel of the harmonic oscillator between $q$ successive quantum levels, say from the $n+q$ level to $n$ level, requires $q$ intervals required for transitions between $n+1$ and $n$ :

$$
\Delta t_{q}=q \Delta t_{1}=q T .
$$

By taking into account that the energy change between $n+q$ and $n$ is

$$
E_{n+q}-E_{n}=q \Delta E=q h v,
$$

where $\Delta E$ is given in (45), we obtain

$$
\frac{q \Delta E}{\Delta t_{q}}=\frac{q h v}{q T}=\frac{h v}{T}=h v^{2}
$$

for all emission rates of the harmonic oscillator.

An evident consequence of (58) is that product

$$
\left(q \Delta E / \Delta t_{q}\right) T^{2}=h
$$

holds for any emission rate entering (58).

\section{Conclusions}

A semiclassical examination of properties of the emission intensity has been done, first, for the energy quanta of a free-electron particle enclosed in a linear potential box, next for the spectrum of a harmonic oscillator. Different separations between the beginning and end quantum levels involved in the emission process are taken into account. The calculations have been performed on the basis of a semiclassical Joule-Lenz law adapted to examination of the quantum systems.

In the first step it is found that the emission rate for a free particle is dependent on the box length $L$ and the index $n$ of the quantum state involved in emission, but not on a mutual separation of the beginning and end quantum levels. This property holds especially well when $n$ is a large number.

On the other hand, in the case of the harmonic oscillator, the emission rate of energy does depend neither on the separation between the states involved in emission, nor on the index $n$ of the quantum level associated with these states. Certainly no selection rules for transitions between the states known from the 
quantum mechanics do apply in the examined semiclassical theory.

A characteristic point is that the detected behaviour of the emission intensity for a free particle and harmonic oscillator is qualitatively different than obtained in the case of the hydrogen atom [8] [9] [10]. In the hydrogen case, the semiclassical emission rate depends essentially on both quantum numbers $n^{\prime}$ and $n$ labelling respectively the beginning and end state of emission.

However, there exists a property which is common for a semiclassical emission examined in all three physical cases mentioned above [3] [10] [11]. It concerns the intensity of emission done between two neighbouring quantum levels, say $n+1$ and $n$; here $n$ should be not a very small integer number. When $\Delta E$ is the energy interval between states $n+1$ and $n$, and the time interval necessary to perform the transition from $n+1$ to $n$ is $\Delta t$, it satisfied the relation

$$
\Delta E \Delta t=h .
$$

Therefore

$$
\frac{1}{\Delta t}=\frac{\Delta E}{h}
$$

and

$$
\frac{\Delta E}{\Delta t}=\frac{(\Delta E)^{2}}{h} .
$$

It is easy to check that the above properties provide us with the emission behaviour for a free particle as well as harmonic oscillator equal to that obtained in the present paper. For a free electron (62) gives with the aid of (15), (14) and (17):

$$
\frac{\Delta E}{\Delta t}=\frac{n^{2} h^{3}}{16\left(m L^{2}\right)^{2}}
$$

which is a result identical with the formulae (16), (23), (28), (32) and (41) attained in Secs. 4 and 5.

In the case of the harmonic oscillator (62) becomes

$$
\frac{\Delta E}{\Delta t}=h v^{2}
$$

which is identical with the expressions presented in (51) and (58).

Let us note that the choice of $\Delta E$ equal to the energy distance between two neighbouring quantum states is in agreement with the well-known quantummechanical selection rule allowing for the dipole radiation of the harmonic oscillator.

\section{Conflicts of Interest}

The authors declare no conflicts of interest regarding the publication of this paper. 


\section{References}

[1] Lass, H. (1950) Vector and Tensor Analysis. McGraw-Hill, New York.

[2] Matveev, A.N. (1964) Electrodynamics and the Theory of Relativity, Izd. Wyzszaja Szkola, Moscow. (In Russian)

[3] Olszewski, S. (2015) Non-Probabilistic Approach to the Time of Energy Emission in Small Quantum Systems. Journal of Modern Physics, 6, 1277-1288. https://doi.org/10.4236/jmp.2015.69133

[4] Eyring, H. and Walter, J. and Kimball, G.E. (1957) Quantum Chemistry. Wiley, New York.

[5] Slater, J.C. (1960) Quantum Theory of the Atomic Structure. McGraw-Hill, New York.

[6] Schiff, L.I. (1968) Quantum Mechanics. 3rd Edition, McGraw-Hill, New York.

[7] MacDonald, A.H. (1989) Quantum Hall Effect-A Perspective. Kluwer, Milano. https://doi.org/10.1007/978-94-010-9709-3

[8] Olszewski, S. (2016) Emission Intensity in the Hydrogen Atom Calculated from a Non-Probabilistic Approach to the Electron Transitions. Journal of Modern Physics, 7, 827-851. https://doi.org/10.4236/jmp.2016.78076

[9] Olszewski, S. (2016) Semiclassical and Quantum-Mechanical Formalism Applied in Calculating the Emission Intensity of the Atomic Hydrogen. Journal of Modern Physics, 7, 1004-1020. https://doi.org/10.4236/jmp.2016.79091

(2016) Erratum to "Semiclassical and Quantum-Mechanical Formalism Applied in Calculating the Emission Intensity of the Atomic Hydrogen" [Journal of Modern Physics 7 (2016) 1004-1020]. Journal of Modern Physics, 7, 2314-2315. https://doi.org/10.4236/jmp.2016.716199

[10] Olszewski, S. (2017) Time Intervals of the Electron Transitions and Intensity Spectrum of the Hydrogen Atom. Journal of Computational and Theoretical Nanoscience, 14, 4086-4099. https://doi.org/10.1166/jctn.2017.6791

[11] Olszewski, S. (2016) Quantum Aspects of the Joule-Lenz Law. Journal of Modern Physics, 7, 162-174. https://doi.org/10.4236/jmp.2016.71018 